\title{
The directionality of topological explanations
}

\author{
Daniel Kostić ${ }^{1}$ (D) $\cdot$ Kareem Khalifa ${ }^{2}$
}

Received: 25 January 2021 / Accepted: 9 September 2021 / Published online: 1 November 2021

(C) The Author(s) 2021

\begin{abstract}
Proponents of ontic conceptions of explanation require all explanations to be backed by causal, constitutive, or similar relations. Among their justifications is that only ontic conceptions can do justice to the 'directionality' of explanation, i.e., the requirement that if $X$ explains $Y$, then not- $Y$ does not explain not- $X$. Using topological explanations as an illustration, we argue that non-ontic conceptions of explanation have ample resources for securing the directionality of explanations. The different ways in which neuroscientists rely on multiplexes involving both functional and anatomical connectivity in their topological explanations vividly illustrate why ontic considerations are frequently (if not always) irrelevant to explanatory directionality. Therefore, directionality poses no problem to non-ontic conceptions of explanation.
\end{abstract}

Keywords Explanation - Directionality · Multiplexes · Topological explanation · Ontic conceptions of explanation $\cdot$ Neuroscience

\section{Introduction}

Philosophical discussions about scientific explanation are guided by two powerful intuitions. The first is that scientific explanations ought to capture the world's structure. The second is that scientific explanations provide understanding. The two intuitions are often in lockstep, but they occasionally come apart—especially when the mathematical resources of science's best explanatory models outstrip scientists' capacity to map

This article belongs to the topical collection "Multiplexes and mechanisms in neuroscience and psychiatry", edited by Leon de Bruin, Beate Krickel, and Linda Douw.

Daniel Kostić

daniel.kostic@gmail.com

Kareem Khalifa

kkhalifa@middlebury.edu

1 Radboud Excellence Initiative Fellow, Institute for Science in Society (ISiS), Radboud University, Huygens Building, Heyendaalseweg 135, 6525 AJ Nijmegen, The Netherlands

2 Middlebury College, Twilight Hall 303A, Middlebury, VT 05753, USA 
them neatly onto the dependency relations that make up its worldly structure. In such cases, explanations seem to work without an ontological net.

In response to these cases, so-called ontic theorists hold fast to the first intuition and provide a litany of challenges to those who wander from their flock. This is especially clear in discussions of so-called topological or "network" explanations. While defenders of topological explanation have addressed some of these challenges, e.g. asymmetry (Kostić, 2020), other challenges remain. In several papers, Craver and Povich pose a distinctive "directionality problem" for mathematical explanations, including some topological explanations (Craver, 2016; Craver \& Povich, 2017; Povich, 2019, 2020; Povich \& Craver, 2018). Roughly stated, causal explanations are directional in the sense that causes explain effects but the absence of effects typically does not explain the absence of causes. Craver and Povich argue that without causality or some other kind of "ontic dependence relation," mathematical explanations in general-and topological explanations in particular-lack an analogous kind of directionality.

In this paper, we provide a non-ontic account of topological explanations' directionality. In so doing, we thereby answer Craver and Povich's challenge, by showing that directionality poses no serious threat to those who do not drink from the ontic well. Furthermore, we highlight the importance of topological explanations involving multiplexes in evaluating different solutions to the directionality problem. Specifically, we will argue that if ontic theorists are correct, these explanations can only be directional under a far narrower set of conditions than neuroscientific practice allows.

We shall proceed as follows. First, we characterize the directionality problem with greater precision than our predecessors. We will argue that when the directionality problem is stated abstractly, it seems like a very serious problem (Sect. 2). However, using the case of topological explanations as our guide, we show that the seriousness of the directionality problem evaporates under closer scrutiny. A more detailed account of topological explanation (as we provide in Sect. 3), paired with examples more reflective of scientific practice, reveals that an overwhelming number of topological explanations in science exhibit the required directionality without appeals to any ontic dependence relations (Sects. 4 through 6). By the end of our romp, we will see that the basis for generating directionality problems is a rather narrow sliver of cases that are little more than intellectual curiosities. Hence, we conclude that those who distance themselves from ontic conceptions of explanation have ample resources for addressing the most serious of directionality problems.

\section{The directionality problem}

As we noted, Craver and Povich first put their finger on the directionality problem. However, its precise contours-as well as its distinctiveness-have not been made explicit. Filling this gap in the literature is our first task. (We stress at the outset that we are more interested in formulating an interesting problem concerning explanation than we are in Craver and Povich exegesis.) Sect. 2.1 provides both a formal and an intuitive statement of the directionality problem. For further clarification, Sect. 2.2 contrasts the directionality problem with the more venerable asymmetry problem, 


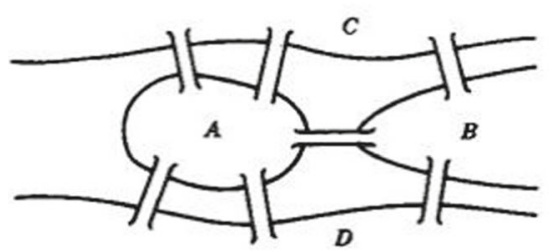

(a) Kőnigsberg in 1736

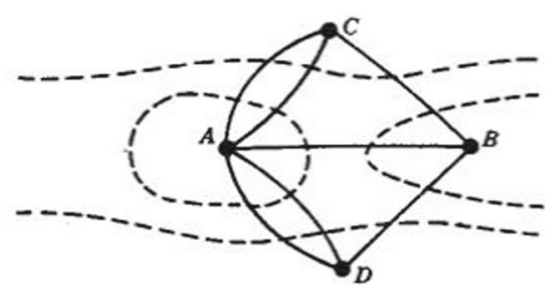

(b) Euler's graphical representation

Fig. 1 Euler's representation of Königsberg's bridges in 1736

arguing that the former's novelty should not obscure the greater threat that it appears to pose to non-ontic conceptions of explanation.

\subsection{What is directionality? Why is it a problem?}

At root, the directionality problem is an objection posed to proponents of non-ontic conceptions of explanation. ${ }^{1}$ For the purposes of this paper, a conception of explanation is ontic if it requires every explanatory relation to be (or accurately represent) ${ }^{2}$ an "ontic dependency relation," which we will take to be causal, constitutive, or nomic (Craver \& Povich, 2017, p. 32). ${ }^{3}$ Thus, non-ontic conceptions of explanation claim that explanatory relations need not be one of these ontic dependency relations. Furthermore, insofar as explanations support counterfactuals (as we require below), ontic theorists demand that these relations "back them;" to serve as the counterfactuals' truth-makers (Craver \& Povich, 2017, p. 37: n. 15). As Povich (2019, p. 26) puts it, "Relations of counterfactual dependence hold in virtue of relations of ontic dependence."

So, what is the precise challenge that directionality poses to non-ontic conceptions of explanation? Here it is in a nutshell. The following seems true of all explanations:

If $X$ explains $Y$, then not- $Y^{\prime}$ does not explain not- $X^{\prime}$, where $X$ and $Y$ are highly similar but not identical to $X^{\prime}$ and $Y^{\prime}$ respectively.

For instance, Jack's smoking explains why he has lung cancer, but Jill's lack of lung cancer doesn't explain why she doesn't smoke. The directionality problem arises for non-ontic theorists of explanation, who seem unable to meet this requirement in certain cases.

This is most immediately appreciated by a shopworn example. Consider the geography of Königsberg in 1736 (Fig. 1). Mathematician Leonard Euler wondered why it is impossible to traverse the city crossing every bridge exactly once-an instance of

\footnotetext{
${ }^{1}$ Discussions thus far focus exclusively on directionality problems for distinctively mathematical explanations. However, for reasons we discuss below, the directionality problem is in fact much broader.

2 This parenthetical is sometimes contested as abdicating everything to ontic theorists' main opponents-socalled epistemic theorists of explanation (Wright 2015). However, as has been noted by many, Salmon (1989, p. 86), the chief progenitor of (or culprit behind?) this distinction took both ontic dependency relations and their representations to be available to ontic theorists. Since Salmon's way of carving up the problem space better suits this paper's purposes, we will switch freely between these two ways of talking.

3 This list is sometimes expanded to include other relations; we discuss this below.
} 
Table 1 Example of the Directionality Problem

\begin{tabular}{lll}
\hline Mathematical & Original & Reversal \\
\hline $\begin{array}{l}\text { Premise } \\
\text { Empirical Premise }\end{array}$ & $\begin{array}{c}\text { A connected graph has neither zero nor } \\
\text { two nodes of odd degree if and only if } \\
\text { an Eulerian path around that graph is } \\
\text { impossible }\end{array}$ & $\begin{array}{c}\text { A connected graph has neither zero } \\
\text { nor two nodes of odd degree if and } \\
\text { only if an Eulerian path around that } \\
\text { graph is impossible }\end{array}$ \\
$\begin{array}{c}\text { neither zero nor two landmasses } \\
\text { connected by an odd number of bridges }\end{array}$ & $\begin{array}{c}\text { An Eulerian path around Kaliningrad } \\
\text { (modern-day Königsberg) is } \\
\text { possible }\end{array}$ \\
in 1736 was impossible & $\begin{array}{c}\text { So, the bridges of Kaliningrad have } \\
\text { either zero or two landmasses } \\
\text { connected by an odd number of } \\
\text { bridges }\end{array}$ \\
\hline
\end{tabular}

what has since been dubbed an "Eulerian path." Representing Königsberg's bridgelandmass structure as a graph, Euler noted that his eponymous paths are possible only for connected graphs with zero or two nodes of odd degree. Since all four of Königsberg's landmasses were connected by an odd number of bridges, it follows that Königsberg cannot be traversed by crossing each bridge exactly once.

To set up the directionality problem, it will be useful to retell a bit more of this city's subsequent history. Two of Königsberg's bridges from Euler's time were subsequently destroyed during World War II. Two others were demolished and replaced by a single highway. Two additional bridges were built, but they connect different landmasses than in Euler's day. ${ }^{4}$ Crucially, in modern-day Königsberg-now called Kaliningrad - two landmasses have an even number of bridges, and two have an odd number of bridges. Thus, unlike Euler's Königsberg, Kaliningrad's bridge-landmass structure forms a connected network with four nodes containing an Eulerian path. Hereafter, "Königsberg" refers to the city as it was in 1736; "Kaliningrad," as it is in 2021.

To appreciate the directionality problem, consider the two inferences in Table 1. The first of these represents Euler's reasoning and is an explanation-what we call the "original." Intuitively, the second, its "reversal," is not an explanation. This is precisely as the directionality requirement prescribes.

If this example begets a directionality problem, then ontic conceptions of explanation should correctly classify the original as an explanation and the reversal as a non-explanation. By contrast, non-ontic conceptions should classify both the original and the reversal as explanations. This requires ontic theorists to provide a constructive argument that their theories can establish this desired classification and also a negative argument that other accounts of explanation cannot. We rehearse each in turn.

Regarding the constructive argument, ontic theories can handle this example in at least two ways. First, landmasses and bridges are parts of a city that are spatially organized, and can be intervened upon with, e.g., explosives, bridge-building, and

\footnotetext{
4 If one looks at the map of Königsberg in Fig. 1, then in modern-day Kaliningrad, landmass $A$ has three bridges; $B$, five; $C$, two; and $D$, four. At least this is our best guess from squinting at Google Maps.
} 
artificial islands. However, there is no clear way of intervening upon an Eulerian path (Elber-Dorozko, 2018). Given that intervention and causation are frequently thought to go hand in hand, the ontic theorist may claim that causal relations account for this example's directionality.

Alternatively, instead of causation, ontic theorists may argue that constitution accounts for this example's directionality. This treatment of the Königsberg example appeals to the intuition that an Eulerian path is constituted by the city's bridges and landmasses. Hence, Euler's reasoning appears to satisfy the ontic conception. However, for parallel reasons, the Kaliningrad reversal does not: since bridges and landmasses constitute the Eulerian path, bridges and landmasses cannot be constituted by the Eulerian path (Craver, 2016; Craver \& Povich, 2017).

Of course, the directionality problem only has teeth if non-ontic conceptions of explanations cannot preserve the difference between good explanations and their reversals. This brings us to the ontic theorist's negative argument. Two non-ontic approaches' failures to solve the directionality problem make this negative argument vivid. $^{5}$

Consider Lange's $(2013,2017)$ modal view, in which explanations are not characterized in terms of ontic dependency relations, but rather in terms of the kind of necessity that they confer upon their explananda. Lange takes Euler's original Königsberg explanation to be an example of a modal explanation: it shows why an Eulerian path was impossible. This is because the major premise of Euler's explanation is a mathematical statement and thereby confers mathematical necessity upon its conclusion. However, by parity of reasoning, the "Kaliningrad reversal" confers the same high-grade necessity upon its conclusion, but it's not an explanation. Hence, modal considerations alone do not solve the directionality problem. By contrast, we have already seen that ontic considerations alone readily solve the directionality problem. ${ }^{6}$

Similarly, several non-ontic counterfactual accounts of the Königsberg bridge example have been proposed (Jansson \& Saatsi, 2017; Reutlinger, 2016, 2018; Woodward, 2018). ${ }^{7}$ These views require the explanandum to counterfactually vary with the explanans, but do not require it to be backed by an ontic dependency relation. On such views, if $X$ explains $Y$, then, had $X$ been different, $Y$ would have been different. In the Königsberg example, the relevant counterfactual would be:

Had Königsberg's bridges formed a connected network containing zero or two nodes with odd degree, then there would have been an Eulerian path around the bridges of Königsberg.

Remove the bridge/edge going from A to B in Fig. 1. Then the antecedent of this counterfactual is true, and with what appears to be a minimal change to the actual structure of Königsberg's bridges ("a nearby possible world”). The consequent

\footnotetext{
5 Povich (2019) argues that another non-ontic view, Baron's (2017) deductive-mathematical account, also fails to solve the directionality problem.

6 To be sure, Lange (2018) has proposed a solution to this problem. However, at least one of us holds that his reply is unsatisfactory for reasons presented by Povich (2020). As such, we will not discuss his views here.

7 Our own position-presented in Sect. 3-is also a non-ontic counterfactual approach. It differs in subtle but important ways from the authors cited above.
} 
of this counterfactual is also true. An Eulerian path would be: DACBDAC. So, the counterfactual theories deliver the right verdict on the original.

However, counterfactual approaches will wrongly classify the Kaliningrad reversal as an explanation. The relevant counterfactual would be:

Had Kaliningrad contained no Eulerian path, then neither zero nor two of its landmasses would have had an odd number of bridges.

This counterfactual must be true on pain of contradicting the mathematical premise shared by both Euler's reasoning and the Kaliningrad reversal. Thus, like Lange's modal conception, counterfactual considerations alone do not solve the directionality problem, and in this way are at a disadvantage in comparison to ontic considerations. However, as we will argue below, our more regimented non-ontic counterfactual account of topological explanation can solve the directionality problem.

\subsection{Directionality and asymmetry}

At this point, readers may be wondering what distinguishes the directionality problem from the better-known asymmetry problem. ${ }^{8}$ Recall Bromberger's (1965) classic example of the latter: from the laws of optics and a tower's height, one may infer the length of the tower's shadow. However, from those same laws and the shadow's length, one may also infer the height of the tower. A popular and intuitive solution to the asymmetry problem appeals to the fact that the tower's height causes the length of the shadow, but the shadow's length does not cause the tower's height. Hence, just as ontic conceptions preserve explanation's directionality in the Königsberg example, they also preserve explanation's asymmetry in the tower example. Given these similarities, this section's first task is to clearly distinguish asymmetry from directionality. Its second task is to show how, in certain sense, directionality problems pose a greater threat to non-ontic theories of explanation than asymmetry problems.

Let us begin by distinguishing these two problems. At the most basic level, the difference between asymmetry and directionality is logical. The asymmetry requirement on explanation is:

If $X$ explains $Y$, then $Y^{\prime}$ does not explain $X^{\prime}$, where $X$ and $Y$ are highly similar or identical to $X^{\prime}$ and $Y^{\prime}$ respectively.

By contrast, we defined the directionality requirement on explanation as:

If $X$ explains $Y$, then not- $Y^{\prime}$ does not explain not- $X^{\prime}$, where $X$ and $Y$ are highly similar but not identical to $X^{\prime}$ and $Y^{\prime}$ respectively.

\footnotetext{
8 This concern may be abetted by the fact that earlier philosophical work on explanation is uneven in how it interprets the phrases "directionality" and "asymmetry:" (1) Barrantes (2019), Beni (2019), Elber-Dorozko (2018), and Jansson (2020) treat these words as synonyms. By contrast, on a single page, Craver and Povich $(2017,33)$ vacillate between glossing "directionality" as: (2) a genus that includes, but is not limited to asymmetry, when they say that the tower and shadow "example demonstrates that at least some (and in fact, many) explanations have a preferred direction;" and (3) as something distinct from asymmetry, e.g. "In what follows, we emphasize the directionality of explanations, not their asymmetry." Our definition of directionality accords best with this last reading.
} 
Table 2 Example of how asymmetry entails directionality

\begin{tabular}{llll}
\hline & Original & $\begin{array}{l}\text { Reversal (for } \\
\text { Asymmetry) }\end{array}$ & $\begin{array}{l}\text { Reversal (for } \\
\text { Directionality) }\end{array}$ \\
\hline $\begin{array}{l}\text { Major Premise } \\
\text { Minor Premise }\end{array}$ & $\begin{array}{l}\tan \theta=\text { height/length } \\
\text { Tower } a \text { 's height is } 443 \mathrm{~m}\end{array}$ & $\begin{array}{c}\tan \theta=\text { height/length } \\
\text { Tower } a \text { 's shadow's } \\
\text { length is } 256 \mathrm{~m}\end{array}$ & $\begin{array}{c}\tan \theta=\text { height/length } \\
\text { Tower } b \text { 's shadow's } \\
\text { length is not } 256 \mathrm{~m}\end{array}$ \\
Conclusion & So, its shadow's length is & $\begin{array}{c}\text { So, Tower } a \text { 's height is } \\
\text { 256 } \mathrm{m}\end{array}$ & $\begin{array}{c}\text { So, Tower } b \text { 's height } \\
\text { is not } 443 \mathrm{~m}\end{array}$ \\
\hline
\end{tabular}

We assume that the sun's angle of incidence $\theta=60^{\circ}$ in all three cases

Table 3 Example of directionality without asymmetry

\begin{tabular}{llll}
\hline & Original & Reversal (for Asymmetry) & $\begin{array}{l}\text { Reversal (for } \\
\text { Directionality) }\end{array}$ \\
\hline $\begin{array}{l}\text { Major Premise } \\
\text { Minor Premise }\end{array}$ & $\begin{array}{c}\text { 23 is not divisible by } 3 \\
\text { Mother } a \text { has } 23 \\
\text { strawberries }\end{array}$ & $\begin{array}{c}\text { 23 is not divisible by } 3 \\
\text { Mother } a \text { cannot divide } \\
\text { her strawberries evenly } \\
\text { among her three children }\end{array}$ & $\begin{array}{c}23 \text { is not divisible by } 3 \\
\text { Mother } b \text { can divide her } \\
\text { strawberries evenly } \\
\text { among her three } \\
\text { children }\end{array}$ \\
Conclusion & $\begin{array}{c}\text { Mother } a \text { cannot divide the } \\
\text { strawberries evenly } \\
\text { among her three children }\end{array}$ & $\begin{array}{c}\text { Mother } a \text { has } 23 \\
\text { strawberries }\end{array}$ & $\begin{array}{c}\text { Mother } b \text { does not have } \\
23 \text { strawberries }\end{array}$ \\
\hline
\end{tabular}

Table 2 presents these differences with respect to the tower and shadow problem.

Let us now turn to why directionality problems pose a deeper challenge to nonontic views than asymmetry problems. At root, the idea is that it is "easier" to generate directionality problems than asymmetry problems. More precisely, every instance of an asymmetry problem can be converted to a directionality problem, but not vice versa. In saying this, we recognize that there may be peculiarities about specific cases that make the corresponding directionality problem easy (indeed perhaps trivial) to solve. Indeed, one can see the arguments in Sect. 4 through 6 as vindicating this point. So, it might be more apt to say that every asymmetry problem has a potential directionality problem, but not vice versa. This is implied hereafter.

Begin with the claim that any example of an asymmetry problem will have a corresponding directionality problem. As Table 2 shows, the classic asymmetry problem has a corresponding directionality problem. Clearly, there can be two towers, $a$ and $b$, that make all three inferences in Table 2 sound. This point generalizes (see Appendix).

Turn now to the claim that not every directionality problem has a corresponding asymmetry problem. ${ }^{9}$ For instance, Table 3 's first and third columns present a directionality problem that Craver and Povich (2017) pose to Lange (2013). Like the tower

\footnotetext{
9 The Königsberg example obscures this point, since it can be used as either a directionality or an asymmetry problem (see Appendix). Both Barrantes (2019) and Jansson (2020) discuss this example's asymmetry problem, though neither distinguish directionality from asymmetry.
} 
example, the reversal for directionality (third column) is a valid inference. ${ }^{10}$ As such, there is a directionality problem. However, unlike the tower example, the reversal for asymmetry (second column) is not a valid inference: the conclusion does not follow from the premises. Since the original (first column) is valid and its reversal for asymmetry (second column) is not, the explanation in this particular example exhibits the desired asymmetry. More generally, this establishes that some directionality problems have no corresponding asymmetry problem.

Thus, every instance of an asymmetry problem can be converted to a directionality problem, but not vice versa. As such, we claim that directionality problems are easier to generate than asymmetry problems. This seems like bad news for non-ontic theories of explanation, since it suggests that their critics will have greater freedom to construct different kinds of counterexamples than they would for asymmetry problems. Hence, for non-ontic theorists of explanation, the directionality problem appears more pressing than the asymmetry problem.

\section{From bridges to brains}

Despite this apparent urgency, there is something amiss with the directionality problem. We offer the first of two diagnoses here: no detailed non-ontic account of explanation is used when saddling such accounts with directionality problems. We will argue that in a great many cases, these details prevent directionality from arising.

For the purposes of this essay, we focus on topological or "network" explanations, but many of the lessons we draw here can be generalized to other non-ontic accounts of explanation. To that end, we advance the following theory of topological explanation to probe different ways in which topological explanations avoid directionality problems:

(TE) $a$ 's being $F$ topologically explains why $b$ is $G$ if and only if:

- Veridicality Requirement: $a$ is $F$ and $b$ is $G$ (or approximately so);

- Property Requirement: $F$ is a topological property and $G$ is an empirical property; and

- Counterfactual Requirement: Had $a$ been $F^{\prime}$ (rather than $F$ ), then $b$ would have been $G^{\prime}$ (rather than $G$ );

- Perspectival Requirement: That $a$ is $F$ answers the relevant explanation-seeking question $Q$ about $b$ 's being $G .^{11}$

TE has the benefit of already being a relatively developed account of topological explanation. It draws heavily on Kostić (2020) and Kostić and Khalifa (manuscript). It is also similar in spirit to the other non-ontic counterfactual accounts of explanation mentioned above (Jansson \& Saatsi, 2017; Reutlinger, 2016, 2018; Woodward, 2018), but is more precisely tailored to topological explanation. Section 3.1 discusses its details in further depth.

TE also provides proponents of topological explanation with the needed resources to put the directionality problem to bed. Specifically, Sect. 4 shows how the property

\footnotetext{
${ }^{10}$ Undoubtedly two mothers, $a$ and $b$, have had different numbers of strawberries to distribute evenly among their children, so the inference is sound.

11 In many topological explanations, $a$ and $b$ will be identical.
} 
requirement ensures the directionality of many topological explanations without any appeal to ontic dependence relations. Sections 5 and 6 do the same with the counterfactual and perspectival requirements, respectively.

Our second diagnosis of why the directionality problem misses its mark concerns ontic theorists' choice of examples. Scientific explanations have greater resources to block directionality problems than the Königsberg example suggests. It is in this context that topological explanations in neuroscience which appeal to multiplexes of functional connectivity (FC) and anatomical connectivity (AC) provide an especially fertile set of cases for evaluating solutions to the directionality problem. ${ }^{12} \mathrm{We}$ discuss two such examples and highlight the more general utility of these FC-AC multiplexes in Sects. 4 and 5.

\subsection{Topological explanation: a philosophical account}

Let us discuss each of TE's four conditions in greater detail, using Helling, Petkov, and Kalitzin's (2019) studies of multistate brain networks' effects on epilepsy as an illustration. Their leading hypothesis is that changes in mean functional connectivity (MFC) explain the dynamics of the onset of epileptic seizure or ictal transitions. They conducted prospective studies involving subjects with focal seizures either starting treatment with an anti-epileptic drug or undergoing drug tapering over several days. Helling et al. found that MFC decreased for those who responded positively to their drug treatment and increased for those who responded negatively. Qualitatively speaking, ictogenicity's (the probability of a seizure) dependence on MFC suggests that "oversynchronization" of the brain explains seizures (Kalitzin et al., 2019, 7).

With these details in hand, let us illustrate how this explanation satisfies TE's conditions. Begin with the veridicality requirement, which holds that the explanans and explanandum must be approximately true. Helling et al.'s explanans is an approximately true description of the functional connectivity in each patient's brain. Similarly, in characterizing their explanandum, they accurately describe different patients' ictogenicity plotted against their dosage levels of anti-epileptic medication.

Turn now to TE's second condition, the property requirement. This distinguishes topological explanations from other kinds of explanations. Hence, it is crucial that we define a topological property. Let a predicate be topological if it is properly applied to a graph (or subgraph) and occurs in some nontrivial theorem derived using only mathematical statements, including the characterization of the graph in terms of its vertices and edges. Then a graph's topological predicates denote its corresponding network's topological properties. Paradigmatically, topological properties concern quantifiable patterns of connectivity in a network.

Quite clearly, MFC is a topological property. Importantly, however, it is instantiated in cortical FC networks. Hence, it is not merely some abstract mathematical property that does the explaining. Rather, it is a mathematical property instantiated in a physical system. This requires careful modeling techniques. For instance, the nodes are readings from EEG channels, i.e. the electrodes measuring the brain's electrical

\footnotetext{
12 For the purposes of this paper, we define a FC-AC multiplex as a network made up of interdependent $\mathrm{FC}$ and $\mathrm{AC}$ layers.
} 
activity, and the edges are synchronization likelihoods, which are correlations between pattern recurrences in the time series data generated by two or more EEG channels. The fact that MFC measures synchronization likelihoods across the cortical network underwrites the aforementioned idea of epileptic brains being "oversynchronized."

The property requirement also demands that the explanandum (that $b$ is $G$ ) is an empirical proposition, such that it can serve as a proper object of scientific investigation. Thus, TE is an analysis of topological explanations in the empirical sciences. Insofar as pure mathematics has topological explanations, it is not our concern here. Furthermore, we adopt a broad notion of "empirical" in line with recent work on data, measurement, and phenomena. ${ }^{13}$ For instance, Helling et al.'s explanandum-ictogenicity - is measured in terms of patients' dose-response to anti-epileptic medication.

The counterfactual requirement, mentioned above, differentiates topological explanations from the broader category of topological models. As already mentioned, many hold that explanations' capacity to support change-relating counterfactuals, or answer "what-if-things-had-been-different questions," distinguishes them from other scientific representations. ${ }^{14}$ Topological explanations also answer these questions, but they are distinctive in highlighting counterfactual differences in a system's topological properties. Such counterfactuals can describe what would happen if the system exhibited another topological property (in which case $F^{\prime}$ is contrary to $F$ ) or if it simply lacked its actual topological property (in which case $F^{\prime}$ is contradictory of $F$ ). For instance, Helling et al. are fruitfully interpreted as committed to the following:

Had the patient's MFC decreased (rather than increased), then the patient's ictogenicity would have been lower (rather than its actual level.)

Finally, the perspectival requirement acknowledges that scientists' interests, background knowledge, and presuppositions may favor a particular topological explanation of a phenomenon, even though other topological explanations of the same phenomenon also satisfy the other three requirements. While Achinstein (1983) and van Fraassen (1980) have highlighted general pragmatic aspects of explanation, Kostić (2020) examines the pragmatics specific to topological explanations. For instance, Kostić (2020) notes that some phenomena admit of both "horizontal" topological explanations, in which local topological properties (e.g. properties of subgraphs) are explanatorily central, and "vertical" topological properties, in which a network's global properties are privileged. The perspectival requirement appeals to scientists' contexts to determine which would be the appropriate topological explanation.

\section{Property directionality}

With our account of topological explanation in hand, we will now argue that it entails that topological explanations in science exhibit the desired directionality. The general strategy is this: we consider a correct topological explanation and its reversal, and then

\footnotetext{
13 We are broadly sympathetic to Boyd's (2018) recent account of the empirical.

14 Furthermore, we assume that only non-backtracking counterfactuals underwrite this condition.
} 
show that only the former satisfies TE's four conditions. ${ }^{15}$ Hence, according to TE, the reversal is not an explanation. Furthermore, we do this without appealing to ontic dependence relations. Importantly, the arguments we offer do not conclusively eliminate the possibility of a reversal of a topological explanation. Rather, they drastically contract the space of possible counterexamples. As such, we take the discussion that follows to substantially shift the burden of proof back onto those who would continue to press the directionality objection.

We begin by noting that many topological explanations will be directional because their reversals will not satisfy the property requirement. Consider the general structure of a reversal, that something's being not- $G$ topologically explains why it is not- $F$. Note if "bidirectionality" is to occur, then, per the property requirement, not- $G$ would have to be a topological property. Otherwise, according to TE, the reversal is not a topological explanation. However, this is often not the case. For instance, Helling et al. offer a topological explanation of why seizures are likely to occur in certain patients. If this reversal is an explanation, then ictogenicity must be a topological property. However, it is not. Hence, Helling et al.'s actual explanation satisfies the property requirement, but its reversal does not.

Thus, some topological explanations will be directional because their reversals do not invoke topological properties in their "explanantia." As such, these reversals violate the property requirement. In such cases, we shall say that the explanations are property directional. Note that property directionality rests entirely on the intrinsic properties of the explanans and the explanandum; it does not hinge on the relationship between the explanans and the explanandum. As such, property directionality holds regardless of whether this relationship is an ontic dependency relation. Hence, ontic theorists have no monopoly on the solutions of directionality problems in which only the explanans-property is topological. This constitutes a substantial portion of the topological explanations found in science.

In this example, the original and reversal's difference with respect to the property requirement show that ontic backing is not needed to establish directionality. Parallel points apply to TE's other requirements. Call this the ontic irrelevance lesson. Imparting this lesson is sufficient for our current purposes. However, the researchers' methods for situating their FC model within a more encompassing multiplex involving AC suggest an added bonus: namely, that ontic backing is not needed at all in this example. As Craver $(2016,705)$ notes, functional connectivity models do not represent mechanisms. ${ }^{16}$ Rather, he asserts that the main network-based explanations in neuroscience must appeal to AC. In AC networks, nodes are segregated anatomical regions of the brain (e.g. different Brodmann areas or cortical lobes) and their edges are causal relations. Against Craver's picture of neuroscientific explanation, Helling et al. model the MFC-ictogenicity link in ways that constantly underscore the irrelevance of AC. It is the functional connectivity that does the heavy lifting in their explanation. As such, their use of multiplexes suggests that their explanation requires no ontic backing.

\footnotetext{
15 This strategy mirrors Kostić's (2020) treatment of topological explanations' asymmetry.

16 Craver infers from this that functional connectivity models are not explanatory. However, Kostić (forthcoming) argues against this claim.
} 
First, using a computational or "in silico" model to validate their hypothesis about MFC and ictogenicity, Helling et al. model anatomical connectivity as a random 128node graph with weighted edges and examine how different features of this network affect the link between MFC and ictogenicity. Two broadly "ontic" considerations emerge as potential "backers" of the MFC-ictogenicity link: the connectivity strength of the anatomical network and a local tissue parameter. Crucially, however, the model shows that the MFC-ictogenicity link holds irrespective of whether fluctuations in MFC were driven by changes in either of these two potential ontic backers (Helling, Petkov, and Kalitzin 2019, p. 4).

Furthermore, how they model the AC network provides even further evidence that while these two parameters are ontic, they do not "back" the topological explanation. This in silico model does not represent specific brain regions (e.g. Brodmann's area or specific parts of a lobe), but simply has generic "brain units" as its nodes. As a result of these modeling choices, even the two parameters mentioned above-the anatomical graph's connectivity strength and its local tissue parameter-abstract away too much detail to serve as the truth-makers for the aforementioned counterfactual between MFC and ictogenicity.

All told, then, their modeling strategies appear to entail little more than that the brain has some anatomical structure, though which anatomical structure it has is largely irrelevant to explaining ictogenicity via MFC. By Craver's own lights, ontic dependency relations reside in AC networks. Thus, Helling et al.'s modeling strategies show that functional connectivity has explanatory power independently of ontic backing. So, in addition to the property requirement establishing this explanation's directionality without appealing to any ontic backing, Helling et al.'s use of FC-AC multiplexes casts doubt on the available ontic factors having the capacity to back that explanation in the first place.

\section{Counterfactual directionality}

The previous section showed that the directionality problem only has bite if it involves property bidirectional explanations, i.e. ones in which a system's having one topological property explains why it has another topological property. Interestingly, the Königsberg example exhibits this property bidirectionality, since both Euler's explanation and the Kaliningrad reversal invoke topological properties in their respective "explanantia" (the number of nodes with odd degree and the presence of an Eulerian path, respectively.)

Our previous example of an explanation involving a FC-AC multiplex was property directional, but other explanations of this ilk are property bidirectional. For instance, Adachi et al. (2011) explain why FC is higher than expected in anatomically unconnected areas in macaque cortices by appealing to the frequency of three-node network motifs in the corresponding AC network. ${ }^{17}$ Since connectivity and frequency of network motifs are both topological properties, this explanation, like Euler's and unlike Helling et al.'s, is property bidirectional.

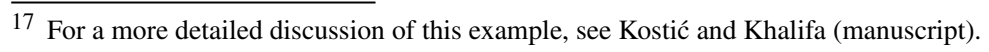


Despite being property bidirectional, explanations involving multilayer networks achieve their directionality through other (non-ontic) means. TE highlights these alternative resources for handling putative directionality problems. Specifically, there will be explanations with originals that satisfy the counterfactual requirement and reversals that don't. Such explanations exhibit counterfactual directionality. Section 5.1 illustrates the power of counterfactual directionality with Adachi et al.'s explanation. Section 5.2 then shows how this example provides valuable lessons for non-ontic treatments of the Königsberg example.

\subsection{Counterfactual directionality in neuroscience}

Adachi et al. show that the FC between any two anatomically unconnected areas in the macaque cortex counterfactually depends on the AC network's overall frequency of three-node network motifs. They establish this by running simulations in which randomly generated networks matched different topological properties of the macaque's AC network, such as global clustering coefficient, modularity, the frequency of two-node motifs, and the frequency of three-node motifs. The last of these-called MF3 - vastly outperformed the others in accounting for changes in the FC found in the macaque neocortex. This suggests the following counterfactual:

(CF1) Had the frequency of three-node motifs (rather than the clustering coefficient, modularity, or frequency of two-node motifs) in the macaque's AC network been lower, then the macaque's FC would have been lower (rather than its actual value). ${ }^{18}$

The reversal of this states that low FC explains low MF3. Consequently, if this reversal satisfies the counterfactual requirement, then the following must be true:

(CF2) Had the macaque's FC been higher (rather than its actual value), then the frequency of three-node motifs (rather than its clustering coefficient, modularity, or frequency of two-node motifs) in the macaque's AC network would have been higher.

Given the contrast class in the consequent, the reversal's counterfactual (CF2) is false. For instance, if FC increases the frequency of three-node network motifs, then it also increases the frequency of two-node network motifs. ${ }^{19}$ However, the consequent is only true if FC increases the frequency of three-node network motifs without also increasing the other topological properties in the contrast class. Consequently, the reversal does not satisfy TE's counterfactual requirement. Since the original does satisfy this requirement, Adachi et al.'s explanation does not beget a directionality problem.

As before, the ontic irrelevancy lesson applies: there is simply no need to look for ontic backing if only the original supports true counterfactuals. Adachi et al.'s statistical tests show that the relevant counterfactual (CF1) is true, and that (CF2) is

\footnotetext{
18 Strictly speaking, the dependent variable (explanandum) is the total increment of FC due to AC patterns of length 2, where the FC is between two areas that do not exhibit a direct AC link (see Fig. 2 for relevant examples). The simplification does not matter for the purposes at hand.

19 The converse of this is not true, so this is not a problem for CF1.
} 


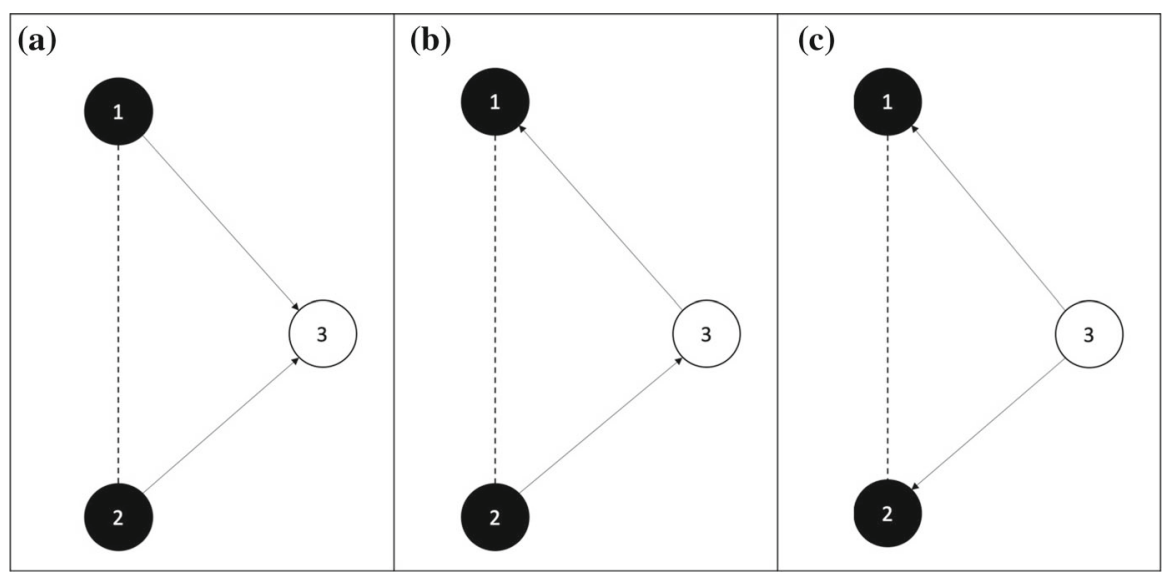

Fig. 2 Different anatomical connectivity patterns $($ length $=2)$ discussed by Adachi et al. (2011). The black nodes, 1 and 2, are functionally connected by the dotted line, but are only indirectly anatomically connected via node 3 by the arrows

false, and that difference alone suffices to capture the directionality of their explanation. Crucially, it does so without saying what makes CF1 true and CF2 false, and is thereby compatible with any workable semantics for counterfactuals-even a semantics that doesn't invoke ontic relations or truth-makers. Once again, this is sufficient for our purposes, yet further exploration of FC-AC multiplexes provides an added bonus: it illustrates how some explanations clearly exhibit counterfactual directionality but do not have a clear ontic backer. For instance, Adachi et al.'s explanation is not backed by nomic, constitutive, or causal relations. It invokes no obvious laws of nature, so nomic relations are implausible ontic backers of the aforementioned counterfactual (CF1). Nor are constitutive relations plausible: the AC and the FC networks have the same brain regions as their nodes. Presumably, when an explanans and explanandum appeal to the same entities at the same level of description, one cannot constitute another.

Adachi et al.'s explanation is also noncausal. Consider two brain areas that are functionally connected (and hence are correlated) but are also known to be anatomically unconnected, i.e., known to lack any direct causal link. In principle, their functional connection could be explained by some indirect cause, such as a third region serving as either an intermediate cause or a common cause of FC between the two regions, as represented by items $b$ and $c$ in Fig. 2. However, Adachi et al. show that some functional connections occur even when two anatomically connected areas only share a common effect (as illustrated by item $a$ in Fig. 2.) Ontic theorists cannot claim that the effect-to-cause relation ontically backs explanations, because doing so would invite a host of asymmetry problems. Hence, no causal interactions in the AC network could ontically back these FC patterns. By contrast, these patterns are explained by the AC network's frequency of three-node motifs - a global topological property. Hence, it is far from obvious whether any ontic backing occurs in this case, whereas its counterfactual directionality is apparent. 
In response, ontic theorists may insist that there are other ontic relations than nomic, constitutive, or causal relations, and it is these other relations that back CF1. However, without precise and general criteria for identifying ontic relations, this looks unmotivated. ${ }^{20}$ For instance, Craver and Povich $(2017$, p. 32) assert that statistical-relevance is an ontic dependency relation. However, in our estimate, if counterfactuals need ontic backing, then so do statistical-relevance relations. Indeed, one might interpret Salmon (1984), the godfather of both ontic and statistical-relevance conceptions of explanation, as conceding as much with respect to his earlier work on statistical relevance (e.g., Salmon, 1971). Furthermore, as regards the directionality problem, the search for ontic backings is superfluous when counterfactual directionality has been established. In these cases, the counterfactuals alone establish the directionality of the explanation. Hence, for theorists of explanation who prefer metaphysical austerity, ontic theorizing can fall by the wayside in such examples.

Moreover, our two examples show how neuroscientists' use of FC-AC multiplexes provide powerful tools for evaluating whether an explanation is ontically backed-and they do so in remarkably different ways. Helling et al.'s explanation shows how neuroscientists sometimes use these multiplexes to establish the "ontic independence" of FC-based explanations. By contrast, Adachi et al.'s explanation shows how even when an explanation is backed by an AC network, it is not thereby ontically backed. All told, then, it appears that ontic conceptions do not do justice to FC-AC multiplexes' diverse explanatory roles.

\subsection{Ontic irrelevance, Königsberg style}

We shall now argue that the ontic irrelevance lesson provides a solution to the directionality problem posed by Königsberg's bridges. Ironically, it is one that we take almost verbatim from Povich (2019). The only difference is that we heed the ontic irrelevance lesson, and Povich does not. The crux of Povich's solution to the Königsberg directionality problem is that:

- A distinctively mathematical explanation (DME) "shows a natural fact weakly necessarily to depend counterfactually only on a mathematical fact" Povich (2019, p. 17). ${ }^{21}$

- In the Königsberg example, the original-but not the reversal—satisfies these requirements for a DME.

Let's unpack these claims in tandem. Povich claims that Euler's original supports the following counterpossible ${ }^{22}$ :

\footnotetext{
${ }^{20}$ Povich's (2018) "generalized ontic conception" is the most explicit on this front, but it merely acknowledges that some ontic dependency relations are noncausal. It does not identify what distinguishes ontic dependency relations from other kinds of relations. Povich (2019, p. 24) outlines two additional ontic relations, the grounding relation and a structuralist-inspired instantiation-realization relation. However, he does not indicate what they share with nomic, constitutive, and causal relations.

${ }^{21}$ Povich has a second route to DME-hood, but it is derivative of this first one. We omit it for simplicity's sake.

22 Mathematical counterpossibles (hence "CP") face formidable semantic difficulties (Nolan, 2013). For the sake of argument, we shall follow Povich in accepting the framework for evaluating counterpossibles proposed by Baron, Colyvan, and Ripley (2017).
} 
(CP1) Had network structure $P$ permitted an Eulerian walk, Königsberg's bridges, which have network structure $P$, would have permitted an Eulerian walk (Povich, 2019, p. 18).

Here, "network structure $P$ " refers to the property of being a four-node network with three nodes having three edges and one node having five, just as in Königsberg. By "weak necessity," Povich (2019, p. 16) means that "any [possible] world where the explanandum exists... is a world where [the counterpossible] is true." Thus, in any possible world, in which Königsberg (in 1736) has network structure $P$, such as our own, CP1 is true. Furthermore CP1's antecedent is a denial of Euler's theorem, which means that this theorem is the only explanans. It is in this sense that DME requires a natural fact - in this case, the possibility of an Eulerian walk around Königsberg- to depend "only on a mathematical fact."

For Povich's solution to work, the Kaliningrad reversal must not satisfy the requirements for a DME. On Povich's approach, the directionality problem arises if the Kaliningrad reversal supports the following counterfactual ${ }^{23}$ :

(CP2) Had network structure $P$ permitted an Eulerian walk, Kaliningrad's bridges, which permit an Eulerian walk, would have had network structure $P$.

Consider our actual world. Kaliningrad's bridge-landmass structure has exactly two nodes of odd degree (i.e., not $P$ ), and permits an Eulerian walk. So, the closest possible world in which CP2's antecedent is true will retain these features. But in such a world, CP2's consequent is false. Hence, CP2 is false in the actual world. So, only the original is a DME, and Povich has thereby provided a solution to Königsberg directionality problem.

Note that Povich's solution is merely an instance of our counterfactual directionality. After all, counterpossibles are, by definition, counterfactuals with impossible antecedents, and only CP1 is true. We have not appealed to any ontic facts, only different truth-values for different counterfactuals. So, the ontic irrelevance lesson applies. We need no ontic backing to ascertain the truth of these counterfactuals. Indeed, although we consider "weak necessity" a logical rather than an ontic feature of an explanation, we did not even need to appeal to it in these cases. CP1 is true and CP2 is false in the actual world, just as TE's counterfactual requirement entails. So, nothing about Povich's solution to the Königsberg directionality problem requires us to add anything to TE.

The ontic irrelevance lesson distinguishes us from Povich. While we accept his solution to the directionality problem, we contend that his further efforts to "demystify" these counterfactuals by appeal to exotic ontic dependency relations, such as "grounding" and "structuralist-inspired instantiation-realization" are not needed for the tasks at hand. Indeed, although we are not especially enthusiastic about this kind of metaphysical speculation, let us grant for the sake of argument that it bears some fruit. That fruit still is unnecessary for solving the directionality problem. That task was completed as soon as CP1 and CP2 were found to have differing truth-values, i.e. as soon as counterfactual directionality was established. That is the ontic irrelevance lesson.

\footnotetext{
23 Povich and Craver do not distinguish between Königsberg and Kaliningrad. See the Appendix, and more specifically n. 26, for why we think this is terminologically unfortunate.
} 
In summary, we have argued that property bidirectional explanations in science are possible. However, this does not yet amount to a victory for ontic theorists, for topological explanations in neuroscience that are based on multiplexes provide powerful examples of property bidirectional explanations that are counterfactually directional. Moreover, we have seen that precisely because these explanations straddle different networks, ontic backing is often far more difficult to ascertain than counterfactual directionality. Hence, it appears that many solutions to otherwise-sticky directionality problems can be achieved on the backs of counterfactuals alone. This makes metaphysical assurances from ontic relations merely recreational, but irrelevant to the solution of the directionality problem.

Finally, we conclude this section by noting that the tandem of counterfactual directionality and the ontic irrelevance lesson drastically transform the burden of proof in debates between counterfactual and ontic theorists. Logically speaking, contraposition is not a valid inference-rule for counterfactual conditionals. In other words, "Had $n o t-Y$ been the case, then $n o t-X$ would have been the case" does not follow from "Had $X$ been the case, then $Y$ would have been the case." 24 Hence, the default assumption should be that any account of explanation that has a counterfactual requirement is directional. Something peculiar about the example at hand—such as the Königsberg example's exceptional reliance on a mathematical necessity - must be established to get the reversal to also meet this requirement. Once again, this greatly contracts the space of directionality problems.

\section{Perspectival directionality}

However, the directionality problem will not die quietly. Neither property directionality nor counterfactual directionality can solve some remaining directionality problems- even some that that still involve Konigsberg's bridges. Indeed, the astute reader may have noticed that Povich's interpretation of the Königsberg example differs in important ways from the counterexample to the counterfactual theorists presented in Sect. 2.1. That latter example involved much simpler counterfactuals than $\mathrm{CP} 1$ and $\mathrm{CP} 2$. We repeat them here:

(OCF) Had Königsberg's bridges formed a connected network containing zero or two nodes with odd degree, then there would have been an Eulerian path around the bridges of Königsberg.

(RCF) Had Kaliningrad contained no Eulerian path, then neither zero nor two of its landmasses would have had an odd number of bridges.

The original supports the first counterfactual (OCF); the reversal, the second (RCF). As we argued above, both of these are true. So, they are "counterfactually bidirectional." Moreover, we have also seen that the Königsberg example exhibits property

\footnotetext{
24 Lewis $(1973,35)$ illustrates this with the following example:

(a) If Boris had gone to the party, Olga would still have gone.

(b) If Olga had not gone, Boris would still not have gone.

Suppose Boris wanted to go, but stayed away to avoid Olga. Then (b) is false. Further suppose that Olga would have been even more excited to attend if Boris had, so (a) is true.
} 
bidirectionality. Hence, we would still seem to have a directionality problem at odds with non-ontic counterfactual theories in general, and TE in particular.

However, TE still has one more trick up its sleeve. Recall that TE's perspectival requirement holds that topological explanations must be answers to the relevant explanation-seeking question. An explanation exhibits perspectival directionality whenever its original satisfies this condition but its reversal does not. We submit that only the original typically satisfies the perspectival requirement. To see why, note that the original is an answer to the following (original) why-question:

(OWQ): Why is there no (rather than some) Eulerian path around the bridges of Königsberg?

By parity of reasoning, the reversal's why-question is:

(RWQ): Why do two (rather than one, three, or all four) of Kaliningrad's landmasses have an odd number of bridges?

A why-question is individuated not just by its surface grammar, which mostly specifies its explanandum, but also by what van Fraassen (1980) calls "relevance relations," which can be thought of as constraints on possible answers. Both the specification of the explanandum (most notably its contrast class) and its relevance relation are determined by a specific inquirers' interests, background knowledge, and perhaps other contextual factors.

With these clarifications in hand, our point is quite simple. In typical contexts in which OWQ is asked, the relevance relation will allow at least one answer to satisfy TE's property requirement. In other words, some statements that appeal to a topological property will be live options as answers. By contrast, in typical contexts in which RWQ is asked, the relevance relation will likely prohibit any topological answers. If someone asked why exactly two of Kaliningrad's landmasses have an odd number of bridges, they would expect some retelling of the city's history of urban planning, but would be sorely disappointed by a math lesson. So, insofar as we are concerned with typical contexts, perspectival directionality is achieved.

This raises a natural question: can't the ontic theorist simply set up an atypical context to generate the desired directionality problem? Before providing our answer, it's important to note how the tide has turned. We have used property directionality and counterfactual directionality to reduce the space of possible directionality problems significantly. Indeed, with the latter, we seem to have a default presumption that topological explanations (according to TE) are directional. Moreover, these were shown to handle real scientific explanations. Arguably, the Königsberg example is toyish by comparison. We also showed that at least one interpretation of the Königsberg example-Povich's interpretation circumscribed by the ontic irrelevance lesson-also exhibits counterfactual directionality. Finally, we have just shown that on a second interpretation of the Königsberg example — involving OWQ and RWQ - perspectival directionality works in typical contexts. Against this backdrop, we think that defenders of topological explanation can safely bite the bullet: the reversal in this very specific interpretation of a toy example in a highly idiosyncratic context is explanatory. After all, nobody should be remotely surprised that atypical contexts call for atypical explanations. 
On this bullet-biting view, both the original and the reversal are technically correct explanations. By "technically correct explanations," we mean that those models that satisfy TE's requirements, but may be bad or unacceptable explanations. (In other words, they are merely technically correct.) As bullet-biters, we take this specific reversal in this fringe case to be technically correct but bad (e.g. lacking in depth, invariance, specificity, and power) regardless of context. Whether it is ever acceptable depends on the extent to which acceptability is context-specific. If acceptability is contextinvariant, then the reversal is unacceptable. If acceptability is context-sensitive, then the reversal is only acceptable in weird contexts. Hence, the bullet bitten is never too big. ${ }^{25}$

Thus, perspectival directionality accounts for even the toughest cases of putatively bidirectional topological explanations when those cases are restricted to typical contexts. We have seen that the ontic theorist's last resort is to advert to atypical contexts. Here, we have suggested that a bullet-biting strategy is the way to go. We have even shown how this bullet-biting strategy is compatible with these nagging remnants of the directionality problem being bad and unacceptable (yet technically correct) explanations.

\section{Conclusion}

The preceding arguments show that the directionality problem looks a lot scarier than it is. When one speaks abstractly of "non-ontic conceptions of explanation" and only considers examples that seem better-suited for mathematics textbooks than for science journals, the directionality problem appears quite formidable. However, things looked quite different once we turned to a developed account of a particular kind of non-ontic explanation — such as our account of topological explanation-and started trying to churn out directionality problems with neuroscientific explanations, with special attention to FC-AC multiplexes. We found that few directionality problems stuck. Some of the problematic "reversals" did not appeal to the properties required of topological explanations. Other reversals did not support the relevant counterfactuals. Finally, many reversals were pragmatically defective from the perspective of their users. The space of possible directionality problems shrank to a few recherche examples, and even these required little in the way of concession. Hence, we conclude that directionality problems are quite manageable to those who would prefer to keep their theories of explanation ontically parsimonious.

Acknowledgements We would like to thank Mark Povich and Carl F. Craver for their valuable and insightful comments on an earlier draft and some early ideas of this paper, respectively.

\footnotetext{
25 Indeed, Craver and Povich seem to treat "good" explanations, "acceptable" explanations, and technically correct explanations as interchangeable (Craver and Povich 2017, p. 32, 33, 34, respectively). But they appear distinct: Newton's mechanical explanations were good and acceptable but false, so not technically correct. Democritus might have accepted bad but technically correct explanations about basic atomic structure. Finally, it may take some testing before a good and technically correct explanation becomes acceptable.
} 
Funding Daniel Kostić would like to acknowledge funding by the Radboud Excellence Initiative. Kareem Khalifa would like to acknowledge funding from the American Council of Learned Society's Burkhardt Fellowship, "Explanation as Inferential Practice."

Open Access This article is licensed under a Creative Commons Attribution 4.0 International License, which permits use, sharing, adaptation, distribution and reproduction in any medium or format, as long as you give appropriate credit to the original author(s) and the source, provide a link to the Creative Commons licence, and indicate if changes were made. The images or other third party material in this article are included in the article's Creative Commons licence, unless indicated otherwise in a credit line to the material. If material is not included in the article's Creative Commons licence and your intended use is not permitted by statutory regulation or exceeds the permitted use, you will need to obtain permission directly from the copyright holder. To view a copy of this licence, visit http://creativecommons.org/licenses/ by $/ 4.0 /$.

\section{Appendix}

In Sect. 2.2, we claimed that every asymmetry problem has a corresponding directionality problem, but not vice versa. Here, we discuss the underlying logic that justifies this result. Recall the preceding definitions:

Asymmetry Requirement: If $X$ explains $Y$, then $Y^{\prime}$ does not explain $X^{\prime}$, where $X$ and $Y$ are highly similar or identical to $X^{\prime}$ and $Y^{\prime}$ respectively.

Directionality Requirement: If $X$ explains $Y$, then $n o t-Y^{\prime}$ does not explain not- $X^{\prime}$, where $X$ and $Y$ are highly similar but not identical to $X^{\prime}$ and $Y^{\prime}$ respectively.

To this, we also note that each of the originals and reversals in Sect. 2 were represented as inferences with a major premise, minor premise, and a conclusion, and that the major premise stayed the same in both the original and the reversal; only the minor premise differed in these two arguments. These points apply to both asymmetry and directionality problems.

This means that the major premise in asymmetry problems must be at least as logically strong as a biconditional, for only this will guarantee that both originals and reversals will be valid arguments. In effect, the validity of both the original and the reversal is guaranteed through the inference-rule sometimes called "biconditional modus ponens:" from " $X$ if and only if $Y$ " and " $X$," infer " $Y$ " and from " $X$ if and only if $Y$ " and " $Y$," infer " $X$ ". When the major premise is weaker than a biconditional, the reversal will be invalid, as is seen in the case of Mother's strawberries in Table 3.

By contrast, the major premise in directionality problems only needs to be at least as logically strong as a material conditional. In effect, the validity of directionality problems' original is guaranteed by (regular) modus ponens; its reversal, by modus tollens. Moreover, the directionality requirement is itself an expression that, unlike material conditionals, explanations do not obey the inference-rule called "contraposition:" from "if $X$, then $Y$ " infer "if not- $Y$, then not- $X$." So, an effective strategy for generating directionality problems is to present examples that use material conditionals!

Finally, note that one may infer a material conditional (as required by directionality problems) from a biconditional (as required by asymmetry problems), but not vice 
versa. Hence, every asymmetry problem has a corresponding directionality problem, but not vice versa.

There is another difference between asymmetry and directionality problems worth flagging. ${ }^{26}$ In directionality problems, the system in the original (denoted by " $a$ " in Table 2 and Table 3) must be similar but not identical to the system in the reversal (denoted by " $b$ "). Asymmetry problems have no such constraint: the systems may be identical or merely similar and still generate an asymmetry problem. We first explain why directionality problems have this feature, and then explain why this does not make them more restrictive than asymmetry problems.

Most theories of explanation require both explanantia and explananda to be (approximately) true. Call this the veridicality requirement. (TE has this requirement.) If one and the same system were used in a directionality problem and the original satisfied this requirement, then it would be logically impossible for the reversal to also satisfy it. Hence, explanatory directionality would simply be a consequence of the veridicality requirement.

Indeed, without the shift from identity to similarity, the veridicality requirement provides a perfectly general "solution" of the directionality problem. Suppose that $X$ explains $Y$ in the original. Then, by the veridicality requirement, $X$ and $Y$ are true. Now consider the reversal of this explanation required by the directionality problem: not- $Y$ explains not- $X$. By the veridicality requirement, this requires $X$ and $Y$ to be false. So, if the veridicality requirement is true, then the directionality problem does not arise for any account of explanation-ontic or otherwise.

This is why we have used modern-day Kaliningrad (" $b$ ") rather than Königsberg in 1736 (" $a$ ") in our reversal. It is similar enough to Königsberg in 1736 to raise the directionality problem, but different enough to avoid the aforementioned inconsistency. Thus, the Kaliningrad reversal satisfies the veridicality requirement.

Notably, asymmetry problems' reversals, but not directionality problems' reversals, can use either identical or similar systems in their reversals. One might think that this undercuts our claim that directionality problems are easier to generate than asymmetry problems. However, this overestimates the difficulty in finding similar systems. Let $F$ and $G$ be the respective "explanans-property" and "explanandum-property" in the original. For instance, in the Königsberg example, the original's explanans-property is having neither zero nor two nodes of odd degree. Its explanandum-property is containing no Eulerian path. The domain is restricted to connected graphs. Directionality problems are possible so long as there is at least one object $a$ in the domain that is both $F$ and $G$, and another object $b$ in the domain that is neither $F$ nor $G$. For most $F$ and $G$, this is an incredibly low bar to clear. For instance, one could simply build a network from arbitrary objects (say dots and lines on a paper) to create the reversal to Euler's reasoning. It needn't be a city. The bar drops even lower if hypothetical or abstract entities are admissible. More importantly, no extant asymmetry problem has explanans- and explanandum-properties that cannot clear this modest similarity

\footnotetext{
26 We flag this largely because Craver and Povich (Craver 2016; Craver and Povich 2017; Povich 2019) systematically present their examples in a manner inconsistent with the basic logical points made here. We think that this is only an infelicity of presentation, since the logical point is clearly one that Craver and Povich would appreciate.
} 
threshold. Hence, directionality problems" "similarity constraint" does not limit their scope to a very significant degree.

\section{References}

Achinstein, P. (1983). The nature of explanation. Oxford University Press.

Adachi, Y., Osada, T., Sporns, O., Watanabe, T., Matsui, T., Miyamoto, K., \& Miyashita, Y. (2011). Functional connectivity between anatomically unconnected areas is shaped by collective network-level effects in the macaque cortex. Cerebral Cortex, 22(7), 1586-1592. https://doi.org/10.1093/cercor/ bhr234

Baron, S. (2017). Mathematical explanation by law. The British Journal for the Philosophy of Science, 70(3), 683-717. https://doi.org/10.1093/bjps/axx062

Baron, S., Colyvan, M., \& Ripley, D. (2017). How mathematics can make a difference. Philosophers Imprint, 17(3), 1-19.

Barrantes, M. (2019). Explanatory information in mathematical explanations of physical phenomena. Australasian Journal of Philosophy. https://doi.org/10.1080/00048402.2019.1675733

Beni, M. D. (2019). New mechanistic philosophy and the scientific prospects of code biology. Biosemiotics, 12(2), 197-211. https://doi.org/10.1007/s12304-019-09360-0

Boyd, N. M. (2018). Evidence enriched. Philosophy of Science, 85(3), 403-421. https://doi. org/10.1086/697747

Bromberger, S. (1965). An approach to explanation. In R. J. Butler (Ed.), Studies in analytical philosophy (pp. 72-105). Blackwell.

Craver, C. F. (2016). The explanatory power of network models. Philosophy of Science, 83(5), 698-709. https://doi.org/10.1086/687856

Craver, C. F., \& Povich, M. (2017). The directionality of distinctively mathematical explanations. Studies in History and Philosophy of Science Part A, 63, 31-38. https://doi.org/10.1016/j.shpsa.2017.04.005

Elber-Dorozko, L. (2018). Manipulation is key: On why non-mechanistic explanations in the cognitive sciences also describe relations of manipulation and control. Synthese, 195(12), 5319-5337.

Helling, R. M., Petkov, G. H. \& Kalitzin, S. N. 2019. "Expert system for pharmacological epilepsy treatment prognosis and optimal medication dose prescription: computational model and clinical application." Proceedings of the 2nd International Conference on Applications of Intelligent Systems, $10.1145 / 3309772.3309775$

Jansson, L. (2020). Network explanations and explanatory directionality. Philosophical Transactions of the Royal Society b: Biological Sciences, 375(1796), 20190318. https://doi.org/10.1098/rstb.2019.0318

Jansson, L., \& Saatsi, J. (2017). Explanatory abstractions. The British Journal for the Philosophy of Science, 70(3), 817-844. https://doi.org/10.1093/bjps/axx016

Kalitzin, S., Petkov, G., Suffczynski, P., Grigorovsky, V., Bardakjian, B. L., Silva, F. L., \& Carlen, P. L. (2019). Epilepsy as a manifestation of a multistate network of oscillatory systems. Neurobiology of Disease, 130, 104488.

Kostić, D. (2020). General theory of topological explanations and explanatory asymmetry. Philosophical Transactions of the Royal Society b: Biological Sciences, 375(1796), 20190321. https://doi. org/10.1098/rstb.2019.0321

Kostić, D. \& Kareem, K. manuscript. "Decoupling topological explanation from mechanisms."

Kostić, D. forthcoming. "Topological explanations, an opinionated appraisal." In Scientific understanding and representation: Mathematical modeling in the life and physical sciences, edited by Kareem Khalifa, Insa Lawler and Elay Shech. London: Routledge.

Lange, M. (2013). What makes a scientific explanation distinctively mathematical? The British Journal for the Philosophy of Science, 64(3), 485-511.

Lange, M. (2017). Because without cause :Non-causal explanation in science and mathematics. Oxford University Press.

Lange, M. (2018). A reply to Craver and Povich on the directionality of distinctively mathematical explanations. Studies in History and Philosophy of Science Part A, 67, 85-88.

Lewis, D. K. (1973). Counterfactuals. Harvard University Press.

Nolan, D. P. (2013). Impossible worlds. Philosophy Compass, 8(4), 360-372. 
Povich, M. (2018). Minimal models and the generalized ontic conception of scientific explanation. The British Journal for the Philosophy of Science, 69(1), 117-137. https://doi.org/10.1093/bjps/axw019

Povich, M. (2019). The Narrow ontic counterfactual account of distinctively mathematical explanation. The British Journal for the Philosophy of Science. https://doi.org/10.1093/bjps/axz008

Povich, M. (2020). Modality and constitution in distinctively mathematical explanations. European Journal for Philosophy of Science, 10(3), 28. https://doi.org/10.1007/s13194-020-00292-y

Povich, M., \& Craver, C. F. (2018). Review of because without cause: non-causal explanations in science and mathematics. The Philosophical Review, 127(3), 422-426.

Reutlinger, A. (2016). Is there a monist theory of causal and noncausal explanations? The counterfactual theory of scientific explanation. Philosophy of Science, 83(5), 733-745. https://doi.org/10.1086/687859

Reutlinger, A. (2018). Extending the counterfactual theory of explanation. In A. Reutlinger \& J. Saatsi (Eds.), Explanation beyond causation: Philosophical perspectives on non-causal explanations (pp. 74-95). Oxford University Pres.

Salmon, W. C. (Ed.). (1971). Statistical explanation \& statistical relevance. University of Pittsburgh Press.

Salmon, W. C. (1984). Scientific explanation and the causal structure of the world. Princeton University Press.

Salmon, W. C. (1989). Four decades of scientific explanation. In P. Kitcher \& W. Salmon (Eds.), Scientific Explanation (pp. 3-219). University of Minnesota Press.

van Fraassen, B. C. (1980). The scientific image. Clarendon Press.

Woodward, J. (2018). Some varieties of non-causal explanation. In Alexander Reutlinger \& Juha Saatsi (Eds.), Explanation beyond causation: Philosophical perspectives on non-causal explanations. Oxford University Press.

Wright, C. (2015). The ontic conception of scientific explanation. Studies in History and Philosophy of Science Part A, 54, 20-30. https://doi.org/10.1016/j.shpsa.2015.06.001

Publisher's Note Springer Nature remains neutral with regard to jurisdictional claims in published maps and institutional affiliations. 